used to direct a systematic literature search. Published evidence was appraised using the GRADE system. Where the literature search identified sufficient evidence, the GDG made a guideline recommendation. Where there was insufficient evidence, the GDG drafted recommendations based on their expert opinion and reviewed these using a formal Delphi consensus process. This was a joint society (BSPED/CCLG) multidisciplinary national endeavour done to NICE methodology and overseen by RCPCH.

Results The literature search identified 568 articles covering the period Jan 1990 -March 2017. The most commonly reported causes of iTPS and iCDI in children are Langerhans Cell Hystiocytosis (LCH), Germ Cell Tumours (GCT) and craniopharingioma (CP). The average prevalence of LCH, GCT and $\mathrm{CP}$ in 11 case series (including 741 patients) is 16\%, $13 \%$ and $12 \%$, respectively. Overall, congenital defects are responsible for $19 \%$ of the iTPS/iCDI cases, whilst infectious diseases (2\%), trauma (1\%) and inflammatory/autoimmune conditions (1\%) rarely occur in children. In $29 \%$ of the cases no aetiology is identified. Causes of pituitary stalk lesions in adults, metastatic tumours and neurosarcoidosis, do not form part of the differential diagnosis in children. What constitutes a TPS is not consistently defined across studies. High quality evidence was lacking for the majority of the clinical questions and two rounds of Delphi consensus were undertaken. A decision-making flowchart has been developed and will accompany the guideline.

Conclusion The likely aetiology of iTPS and iCDI in children differs from that in adults and justifies the development of age appropriate decision making management guidelines to inform best practice nationally. This will form the basis for future audits of practice and outcomes and is intended to improve the quality of care of children and young people with iTPS and iCDI.

\section{G357 NATIONAL UK GUIDELINES FOR THE INVESTIGATION, TREATMENT AND LONG-TERM FOLLOW-UP OF PAEDIATRIC CRANIOPHARYNGIOMA}

\begin{abstract}
${ }^{1,2} \mathrm{HW}$ Gan, ${ }^{3} \mathrm{P}$ Morillon, ${ }^{4} \mathrm{C}$ Mallucci, ${ }^{5} \mathrm{~A}$ Gamble, ${ }^{6} \mathrm{~S}$ Wilne, ${ }^{7} \mathrm{~B}$ Harrison, ${ }^{2} \mathrm{H}$ Spoudeas. ${ }^{1}$ Genetics and Genomic Medicine Programme, University College London Great Ormond Street Institute of Child Health, London, UK; ${ }^{2}$ Department of Paediatric Endocrinology, Great Ormond Street Hospital for Children NHS Foundation Trust, London, UK; ${ }^{3}$ Department of Emergency Medicine, Kingston Hospital NHS Foundation Trust, London, UK; ${ }^{4}$ Department of Paediatric Neurosurgery, Alder Hey Children's NHS Foundation Trust, Liverpool, UK; ${ }^{5}$ Children's Cancer and Leukaemia Group, Leicester, UK; ${ }^{6}$ Nottingham Children's Hospital, Nottingham University Hospitals NHS Trust, Nottingham, UK; ${ }^{7}$ Department of Endocrine Surgery, Sheffield Teaching Hospitals NHS Foundation Trust, Sheffield, UK
\end{abstract}

10.1136/archdischild-2018-rcpch.347

Aims Although rare, craniopharyngiomas are the commonest suprasellar tumour in childhood. Despite high overall survival, children and young people $<19$ years with craniopharyngiomas are at risk of multiple relapses and long-term tumour- and treatment-related neuroendocrine, cognitive and visual morbidity. We sought to provide, for the first time, a national standard for best practice based on currently available evidence for the assessment, treatment and follow-up of paediatric craniopharyngiomas under the auspices of the RCPCH, UK Children's Cancer and Leukaemia Group (CCLG) and the British Society for Paediatric Endocrinology and Diabetes (BSPED).

Methods Clinical questions were formulated based on a PICO (Population, Intervention, Comparison, Outcome) format by a multidisciplinary Guideline Development Group. Systematic searches were conducted via the Ovid MEDLINE (1946-February 2017) and Cochrane Library (2016, Issue 12) databases, identifying 2023 separate research articles. Publications underwent a three-tier filtering process and 300 were reviewed using the GRADE approach. Where recommendations could not be made, a two-stage international Delphi consensus process was conducted.

Results 44 clinical questions were identified, leading to 35 recommendations largely based on low to very low quality evidence. 30 further recommendations achieved $>70 \%$ agreement via the Delphi consensus process. Important highlights include the recommendation that craniopharyngiomas are managed in tertiary paediatric centres with sufficient neuro-oncology, neurosurgery, endocrinology, radiology, pathology and neuropsychology multidisciplinary experience. At diagnosis, tumours should be graded using the 'Paris' grading system and subsequent surgical treatment tailored to avoid hypothalamic damage, with adjuvant upfront radiotherapy being offered where tumour resection is incomplete. Detailed recommendations on the neuroendocrine, ophthalmological and psychological pretreatment assessment of patients and long-term follow-up of survivors are also made, with a review on the safety of growth hormone replacement therapy in this cohort.

Conclusions These guidelines provide the first evidence- and consensus-based national recommendations for the management of paediatric craniopharyngioma, and highlight the need for further research in areas such as the efficacy of proton beam therapy, radiosurgery and intracystic therapies in children, and the management of late effects such as hypothalamic obesity. Through their implementation, we hope to achieve better consistency in the quality of care of such patients and improve long-term quality of survival.

\section{G358 MENTAL HEALTH NEEDS OF LONG TERM SURVIVORS OF CHILDHOOD AND YOUNG ADULT CANCER}

${ }^{1,2,3} \mathrm{AJ}$ Friend, ${ }^{1,3,4} \mathrm{AW}$ Glaser, ${ }^{2,4} \mathrm{RG}$ Feltbower. 'Leeds Institute of Cancer and Pathology, University of Leeds, Leeds, UK; ${ }^{2}$ Division of Epidemiology and Biostatistic, University of Leeds, Leeds, UK; ${ }^{3}$ Department of Paediatric Oncology, Leeds Children's Hospital, Leeds, UK; ${ }^{4}$ Leeds Institute of Data Analytics, University of Leeds, Leeds, UK

\subsection{6/archdischild-2018-rcpch.348}

Background/objectives Survivors of children's and young people's cancer are known to have an increased risk of cognitive difficulties compared to the general population, however less is known about emotional and behavioural problems. A recent systematic review highlighted the lack of consensus regarding the psychiatric needs of these patients. We aimed to further explore the prevalence of psychiatric disorder in long-term survivors of children's and young people's cancer.

Methods Cancer registration records from a regional population-based registry of children's and young people's cancer in Yorkshire were electronically linked with the Hospital Episode Statistics Mental Health and Learning Disabilities Data Set (MHLDDS) covering all admissions in England between 2005 and 2016. The analysis was limited to those aged 0-29 years at diagnosis between 1974 and 2012; we excluded those diagnosed less than 5 years ago to ensure that patients who required psychological support during their acute treatment were not included.

Results We had registry data for 8092 patients who had survived a minimum of 5 years following a diagnosis of 
childhood or young adult cancer. Of these, $4.36 \%$ had contacts recorded on the Mental Health and Learning Disabilities Data Set. Female survivors (5.36\%) were more likely to have mental health contacts than males $(4.51 \%)$, although this was not statistically significant $(\mathrm{p}>0.05)$. Survivors diagnosed between the ages of 15 and $29(6.24 \%)$ were significantly more likely to have mental health contacts than those diagnosed at 14 or younger $(3.78 \% ; \mathrm{p}<0.001)$. Lymphoma survivors $(5.79 \%)$ were more likely to have mental health contacts than leukaemia survivors $(3.74 \% ; \mathrm{p}=0.006)$ or non-CNS solid tumour survivors $(4.39 \%, p=0.001)$ but not CNS tumour survivors $(4.97 \% ; \mathrm{p}>0.05)$.

Discussion The increased number and proportion of patients diagnosed in the 15-24 age bracket who went on to develop mental health problems compared to those aged 0-14 emphasises the unique needs of this cohort. Further work will investigate the risks of mental health contact according to ethnic group, treatment modality and socio-economic status and seek additional mental health consultation data from linked primary care records.

\section{G359(P) CHARACTERISING THE DISEASE PRESENTATIONS OF LCH OVER 10 YR AT A UK PTC}

'AFM Mitchell, 'R Ramanujachar, ${ }^{2} \mathrm{~J}$ Gray, ${ }^{1} \mathrm{G}$ Nicolin, ${ }^{1} \mathrm{U}$ Uparkhar, ${ }^{3} \mathrm{~B}$ Vadgama, ${ }^{1}$ A Cooke. 'Paediatric Oncology, Southampton Childrens Hospital, Southampton, UK; ${ }^{2}$ Paediatric Oncology, University of Southampton, Southampton, UK; ${ }^{3}$ Paediatric Pathology, University Hospital Southampton NHS Foundation Trust, Southampton, UK

\subsection{6/archdischild-2018-repch.349}

Background Langerhans Cell Histiocytosis (LCH) describes a clinical spectrum of disease caused by accumulation of pathologicallangerhan'scells infiltrating tissues. Further understanding regarding the patho-physiology and natural history of langerhans cell histiocytosis has emerged with the understanding of its status as a clonal BRAF stimulated malignancy.

Objectives To characterise the disease presentations of Langerhans Cell Histiocytosis in a UK Principal treatment centre (PTC) over the past 10 years.

Methods Our PTC serves a population of 3.6 million sharing care with 11 regional shared care centres. A retrospective case review was performed for all patients treated for LCH over the last 10 years. Data were collected on demographics, disease location, treatment modality, intervals of remission free outcome and latest evaluation at follow up.

Results 1041 children have been treated for cancerover the past 10 years (1/1/2006-31/12/2016). We identified 45 cases of $\mathrm{LCH}$ from children aged 2 mths to 16 years (median age of 2 years 6 mths) in our database accounting for $4 \%$ of our patients. There were $44 \%$ females and $58 \%$ males. $64 \%$ of cases presented with single-system disease at diagnosis and $36 \%$ with multi-system disease. 4 children were identified as having diabetes insipidus at diagnosis $2.5 \%$.

$33 / 45(73 \%)$ had a biopsy performed consistent with LCH, 10 had no biopsy performed and 2 had a biopsy which was negative but were treated as per $\mathrm{LCH}$ due to typical clinicradiological correlation. 2 cases of neurodegenerative $\mathrm{LCH}$ were identified in this case series.

Multi-system patients have a more challenging course and in our series 10/18 (56\%) went on to have a recurrence and of these 2 patients have had 4 relapses and are still undergoing further therapy.

Conclusions Our centres experience over the past 10 years which shows the full spectrum of presentation from the benign self-limiting single system bony disease to that of extensive multi-system disease leading to secondary HLH. Novel strategies to evaluate and monitor BRAF in blood and urine such as those being employed in the latest international LCH IV trial offer the opportunity to better understand the risk factors for disease severity.

\section{G360(P) A RANDOMISED CONTROLLED TRIAL OF READY TO USE THERAPEUTIC FOOD (RUTF) FOR MODERATE/SEVERE ACUTE MALNOURISHED INDIAN CHILDREN WITH CANCER}

${ }^{1} \mathrm{~S}$ Tandon, ${ }^{2} \mathrm{~B}$ Arora, ${ }^{2} \mathrm{M}$ Prasad, ${ }^{2} \mathrm{G}$ Narula, ${ }^{2} \mathrm{~S}$ Banavali, ${ }^{3} \mathrm{M}$ Gadge, ${ }^{4} \mathrm{~A}$ Jadhav. ${ }^{1}$ Paediatric Haematology/Oncology, Great Ormond Street Hospital, London, UK; ${ }^{2}$ Paediatric Haematology/Oncology, Tata Memorial Centre, Mumbai, India; ${ }^{3}$ Biochemistry, Tata Memorial Centre, Mumbai, India; ${ }^{4}$ Paediatrics, Sion Hospital, Mumbai, India

\subsection{6/archdischild-2018-rcpch.350}

Background Children with cancer are at increased risk of malnutrition.Early nutrition intervention helps to maintain weight, lean body mass, improved treatment tolerance and QOL. RUTF, with higher recovery rates in pilot studies has brought a paradigm shift in the management of malnutrition.This pioneer trial evaluated the effectiveness of RUTF in prevention of malignancy related weight loss, improvement of micro/macronutrient status, treatment tolerance and QOL.

Methods 70 children (5-15 years) with hematolymphoid and solid tumours were enrolled post the appetite test. Randomization into 1:1 using a computerised table and stratification by type of malignancy. Nutritional status (weight, height, BMI, MUAC, TSF), biochemial analysis, DEXA scan, HRQOL, treatment tolerance evaluated at baseline, 6 weeks and 3 months into study and 6 months follow-up for anthropometry and treatment tolerance.

Results Seventy newly diagnosed MAM/SAM children with cancer with median age 9 years (range, 5-15), M:F 3:1 were randomised into RUTF (37) :control(33) arms. Median protein and calorie intake as well as weight gain at 6 weeks $(2.6 \mathrm{~kg}$ vs. $2 \mathrm{~kg}$ ) was higher in the RUTF arm compared to controls on standard dietary care. At 6 weeks there was significant reduction of MAM/SAM children ((16 vs. $23, \mathrm{p}<0.05))$ with increment of lean mass in the RUTF arm vs. controls. Vitamin B12 and folate deficiency (33\%), vitamin $\mathrm{D}(63 \%), 56 \%$ and $96 \%$ had copper and zinc deficiency respectively, which improved in the RUTF arm. Children on RUTF experienced significant reduction in the episodes of febrile neutropenia (18.9\% vs. $30.3 \%, \mathrm{p}=0.06)$, protocol delays $(2.7 \%$ vs $30.3 \%$, $\mathrm{p}<0.05)$, grade $3 / 4$ neutropenia $(40.54 \%$ vs. $66.7 \%, \mathrm{p}<0.05)$, thrombocytopenia $(21.6 \%$ vs. $30.3 \%, \mathrm{p}<0.05)$ and anaemia $(18.9 \%$ vs. $36.36 \%, p>0.05)$ beyond 6 weeks. Mean HRQOL scores were better in the RUTF arm at baseline, 6 weeks and 3 months.

Conclusion RUTF is cost-effective in improving nutritional status resulting in higher weight and lean body mass which translates into improved treatment tolerance and QOL. 